\title{
La disposición a pagar más por productos vinculados a la RSE: evidencia de un análisis conjunto en México
}

\author{
The willingness to pay for cause related products: Empirical evidence from a \\ conjoint analysis in Mexico
}

\author{
Bernardo Amezcua ${ }^{1}$, Arturo Briseño ${ }^{2, *}$, Teresa Ríos ${ }^{3}$ y Edgardo Ayala ${ }^{4}$ \\ ${ }^{1}$ Universidad Autónoma de Coahuila, México \\ ${ }^{2}$ Universidad Autónoma de Tamaulipas, México \\ ${ }^{3}$ Universidad de Monterrey, México \\ ${ }^{4}$ Tecnológico de Monterrey, México
}

Recibido el 13 de febrero del 2016; aceptado el 27 de octubre del 2016

Disponible en Internet el 19 de marzo de 2018

\section{Resumen}

Las campañas de mercadotecnia alineadas a prácticas de Responsabilidad Social Empresarial (RSE) son cada vez más comunes como una estrategia para lograr la diferenciación de marca y la preferencia de los consumidores. Sin embargo, el reto para muchas empresas está en entender como impactan las campañas sociales y ambientales patrocinadas por una marca en la decisión de compra del consumidor y cómo estas campañas generan beneficio económico y social. Considerando el hecho de que los consumidores toman decisiones de compra basándose en varios atributos, utilizamos el análisis conjunto para determinar el impacto de tres atributos: campaña de RSE, marca, y precio en las decisiones de compra. Se recolectó información de una muestra de consumidores mediante una encuesta electrónica y se analizó utilizando un modelo de regresión con datos panel. Con base a esta muestra, se determinaron los efectos directos e indirectos de las campañas de RSE en la preferencia del consumidor y se desarrolló un método para mapear el incremento en las preferencias en su equivalente monetario. Los resultados arrojan que el consumidor está dispuesto a pagar un sobreprecio aproximado de $22 \%$ por productos líderes en la categoría estudiada asociados a campañas con causa social, en comparación a un $10 \%$ en productos menos posicionados. Este efecto no está presente en campañas ambientales. De esta forma, se demuestra que las campañas sociales crean beneficios económicos para la empresa mientras que la sociedad se beneficia de la participación del consumidor en este tipo de campañas.

\footnotetext{
*Autor para correspondencia.

Correo electrónico: abriseno.dca@gmail.com (A. Briseño)

La revisión por pares es responsabilidad de la Universidad Nacional Autónoma de México.
} 
Códigos JEL: M14, M21, M31, D40

Palabras clave: Preferencia de Marca, Responsabilidad Social Empresarial, Sobreprecio, Análisis Conjunto, Descuento Equivalente.

\begin{abstract}
Marketing campaigns aligned with Corporate Social Responsibility (CSR) practices are increasing as a strategy to reach brand differentiation and consumer preference. However, the challenge for many firms is to understand the impact of social and environmental campaigns in consumer's perception and how these campaigns create economic and social value. Knowing that consumers make decisions based on several attributes, we use a conjoint analysis to determine the influence of CSR campaigns, brand, and price over purchase decisions. Data was collected online from a sample of Mexican consumers and was analyzed using panel data. From this sample we evaluate the direct and indirect effects of CSR campaigns in consumer preference, developing a method to map the preference increase in monetary terms. Our results show that consumers are willing to pay a premium price around $22 \%$ for leading brands if their products are associated with social campaigns and $10 \%$ for regular suppliers. This effect is not present in environmental campaigns. We prove that social campaigns create economic benefits for the firm, whereas society benefits from consumers' participation in social campaigns.
\end{abstract}

JEL Classification: M14, M21, M31, D40

Key words: Brand Preference, Corporate Social Responsibility, Price Premium, Conjoint analysis.

\title{
Introducción
}

En los últimos años se ha visto un incremento en la generación de productos y servicios vinculados a la Responsabilidad Social Empresarial (RSE). Las empresas en su búsqueda de ser percibidas como socialmente responsables intentan establecer una conexión con los consumidores a través del desarrollo de campañas de comunicación que resalten áreas como 1) la preservación de recursos no renovables, 2) preservación de la biósfera y 3) creación de una mejor sociedad (Veenhoven, 2008). Esta conexión empresarial generalmente se logra al alinear las campañas de RSE con lo que piensan los consumidores. Por ejemplo, en México, diversas investigaciones de mercado muestran un aumento en el interés por el consumo de productos y servicios sustentables. De manera similar, la TNS Research International México, reporta que el $70 \%$ de los consumidores encuestados estarían dispuestos a pagar un sobreprecio por productos ecológicos, mientras que el $43 \%$ señaló que el cuidado ambiental influye en sus decisiones de compra (TNS, 2011). De igual forma el 95\% de los consumidores europeos consideran que es bueno comprar productos amigables con el ambiente, y el 54\% tiene la intención real de comprar este tipo de productos (TNS, 2013).

En las campañas de mercadotecnia con causa, el proceso más común implica que las empresas contribuyen una cantidad monetaria predeterminada cada vez que el consumidor adquiere un producto de la compañía (Berglind y Nakata, 2005; Varadarajan y Menon, 1988). En tiempos recientes este tipo de campañas se han vuelto una práctica común en los mercados. Por ejemplo datos del estudio realizado por IEG en los Estados Unidos, reportan un crecimiento del $3.7 \%$ en el 2015 alcanzando un gasto en este tipo de campañas de 1,912 millones de dólares americanos (Cause Marketing Forum, 2015) con una tendencia similar a nivel mundial. Edelman (2014) reporta que de acuerdo al IEG, el gasto en la mercadotecnia 
con causa se ha incrementado casi en un $200 \%$ en los últimos diez años y tendrá cada vez más importancia en la construcción de marca (WFA, 2013) y generación de valor para la empresa (Barone, Miyazaqui y Taylor, 2000). Ante la proliferación de este tipo de campañas se hace imprescindible analizar cuales elementos en una oferta de mercadotecnia con causa tienen más impacto en el consumidor en su proceso de decisión de compra.

Un hecho que se ha demostrado en investigaciones anteriores es que los consumidores no evalúan un solo atributo de la oferta al momento de tomar su decisión sino que evalúan un conjunto de ellos (Lusk y Shogren, 2007). Estudios previos han evaluado, por separado, el impacto de algunos atributos del producto como la marca, las certificaciones de calidad, el precio, y las campañas de responsabilidad social en la preferencia del consumidor (Aaker y Keller, 1990; Keller, 1993; Elliot y Cameron, 1994, Barone, Miyazaki y Taylor, 2000; Mohr y Webb, 2005; Van den Brink, Oderken Schöreder y Powels, 2006). Sin embargo, los resultados no han sido concluyentes, pues algunos aseguran que los consumidores compran bajo el criterio tradicional de precio, calidad y familiaridad con la marca (Boulstridge y Carrigan, 2000; Marin, Ruiz y Rubio, 2009) y por motivos personales y dejando de lado las motivaciones de sociales (Beckmann, Chirstensen y Christensen, 2001).

Aún más importante, la literatura no ha contemplado el estudio simultáneo de éstas variables en la intención de compra. En base a lo anterior, el objetivo de esta investigación es comprobar el efecto conjunto que tiene el tipo de campaña de RSE, la marca y el precio en el nivel de preferencia del consumidor. De esta manera podremos concluir qué tipo de campañas son más efectivas para obtener la preferencia del consumidor y su intención de pagar más por un producto relacionado a una causa de RSE.

Los resultados de este estudio comprueban el efecto moderador de la campaña de RSE en la elasticidad de precios y el reconocimiento de marca en un producto con causa. En otras palabras, el consumidor percibe que, cuando paga más por un producto relacionado a una causa, la sociedad, resulta beneficiada con los donativos obtenidos a través de su aportación y del donativo de la empresa. Así mismo, en la categoría de productos de consumo básico (i.e. leche) los resultados de este estudio demuestran que la campaña social aumenta la efectividad de las marcas más reconocidas o líderes en un $22 \%$ y atenúa el impacto negativo de un incremento en el precio.

El presente trabajo de investigación está estructurado en tres secciones, presentando en la primera parte una revisión de la literatura de la RSE y las iniciativas de mercadotecnia relacionadas a la misma donde se establecen las hipótesis de esta investigación. En la segunda sección se detalla la metodología seguida para la recolección de los datos y su procesamiento. En la tercera sección se analizan y presentan los resultados obtenidos a través de la modelación por datos panel. Finalmente se analiza la contribución de manera independiente de cada variable incluida en el modelo, para discutir como el consumidor mexicano se ve influido en su preferencia de compra por los esfuerzos de RSE realizados por las empresas. Así mismo, se sugieren futuras líneas de investigación para conocer con más detalle la interacción de estas variables y para superar algunas limitantes de este estudio.

\section{Revisión de Literatura}

El concepto de RSE ha sido definido por distintos autores y organizaciones de manera diferente, creando una amplia gama de conceptos que no han podido ser unificados (Briseño, 
Lavín, \& García, 2011; Marsden, 2006; McWilliams et al., 2006). Para efectos de este trabajo se tomará el concepto generado por la Unión Europea, a través de su Libro Verde sobre Responsabilidad Social Empresarial. En este documento, la RSE se define como la integración de preocupaciones sociales y ambientales en las operaciones empresariales y en las interacciones de las empresas con los grupos de interés (stakeholders) de forma voluntaria. Es decir, implica tomar decisiones que vayan más allá de los requerimientos mínimos legales con el propósito de atender necesidades sociales (Comisión de las Comunidades Europeas, 2001).

En países con economías en desarrollo, el concepto de RSE se encuentra aún en etapas iniciales en comparación con países desarrollados. Parte de estas diferencias se centran en la relación gobierno-empresa (Vives, 2008) así como en la falta de conocimiento y/o interés de los consumidores en relación a las responsabilidades de la empresa ante la sociedad (Barroso, 2008; Guzmán, Becker-Olsen, y Hill, 2008).

Para identificar las actividades relacionadas a la RSE, Carroll (1991) sugiere un modelo que incorpora las cuatro responsabilidades que una empresa puede tener y las interacciones entre éstas. Carroll clasifica las responsabilidades en cuatro grupos: a) económicas, b) legales, c) éticas y d) filantrópicas. Con esta clasificación, Carroll sugiere que las responsabilidades empresariales no son solamente generar utilidades, si no que existen otras que deben ser atendidas con la misma importancia. Un aspecto a considerar en este concepto es que cada uno de estas responsabilidades puede examinarse desde la perspectiva individual del stakeholder lo que puede generar estrategias de comunicación que no satisfacen las expectativas de los consumidores.

Esta visión más amplia de las responsabilidades empresariales ha permitido generar una mayor atención a todas las actividades que realizan las empresas para alcanzar sus objetivos económicos. La mercadotecnia, como una de estas actividades, ha tenido un papel importante en la inclusión del concepto de RSE. Por ejemplo, Smith y Williams (2011) reconocen la importancia de la mercadotecnia en la toma de decisiones sobre el diseño y comercialización de productos; no sólo desde el punto de vista de generación de utilidades sino también de los efectos negativos, tanto sociales como ambientales, que se pudieran tener en cualquiera de las actividades del negocio.

Dado este reconocimiento, cada vez hay más opiniones que vinculan a la mercadotecnia como una herramienta en la cual la RSE puede encontrar una plataforma idónea para lanzar sus iniciativas (Piercy y Lane, 2009; Smith y Williams, 2011). Por ejemplo, Piercy y Lane (2009) sugieren que el involucramiento en iniciativas de RSE genera ventajas competitivas para las empresas. Para el logro de estas ventajas, la mercadotecnia tiene un papel relevante especialmente en la comunicación de iniciativas sociales y ambientales dada la creciente exigencia social para la apertura y transparencia empresarial (Piercy y Lane, 2009).

En esta relación entre la mercadotecnia y la RSE, un enfoque recurrente ha sido el identificar el impacto que tienen las iniciativas sociales y ambientales en el consumidor para determinar su eficacia. Distintas investigaciones han buscado identificar las reacciones del consumidor durante su proceso de decisión de compra (Sheikh y Beise-Zee, 2011; Tian, Wang y Yang, 2011). Por ejemplo, en su investigación Sheikh y Beise-Zee (2011), encuentran una relación positiva entre las acciones de RSE y la percepción del consumidor. Así mismo, estos investigadores sugieren que las iniciativas sociales y ambientales deben diseñarse en base a las características particulares de los consumidores del producto para generar un mayor impacto. También, Tian, et al. (2011) encuentran evidencia de las reacciones de consumidores ante iniciativas de RSE. En particular, en su investigación encuentran resultados significativos entre 
la percepción del consumidor hacia la empresa (si es realmente responsable con la sociedad o si solo es una fachada) y su intención de compra. Sin embargo, a pesar de estos avances en la literatura, no se ha profundizado en el impacto de los atributos de campañas de RSE de manera conjunta en la intención de compra. Dado que los consumidores evalúan múltiples atributos al comprar productos involucrados en RSE, la literatura solo ha mostrado parcialmente los efectos de la intención de compra.

\section{Marco Teórico}

Petkus y Woodruff (1992) profundizan en el concepto de la RSE descrito en la sección anterior para incluir la idea de que la empresa puede simultáneamente evitar daños derivados de su operación y hacer el bien. En este sentido, Bloom, Hoeffler, Keller y Basurto (2006) definen a los programas de RSE como las iniciativas empresariales para proveer dinero, otros recursos y/o difundir un mensaje de ayuda social, el cual busca crear en la mente del consumidor una asociación entre la causa apoyada y la empresa o una de sus marcas.

Bloom, et al., (2006) identifican diferentes tipos de programas de RSE que la empresa puede apoyar. Uno de ellos es la mercadotecnia con causa, en dónde por cada unidad de producto vendido se dona un porcentaje a una causa. Para estos autores, los beneficios que permiten obtener las campañas de RSE a las empresas son entre otras, la posibilidad de establecer precios mayores, incrementar la participación de mercados, diferenciarse de los competidores, obtener mayor lealtad a la marca y mejorar la reputación de la empresa entre sus stakeholders.

El presente estudio analiza qué tipo de marcas se ven más favorecidas con la utilización de una campaña de RSE en productos de alto involucramiento y de consumo básico/utilitario (i.e. leche) y la efectividad de tres diferentes campañas de RSE (i.e., social, ecológica y contra el maltrato de animales en granjas) sobre la preferencia del consumidor en dichas marcas.

Algunos estudios previos han evaluado el impacto de las campañas de RSE sobre la imagen de la marcas y de las compañías. Otros han hecho estudios comparativos para determinar qué tipo de campañas sociales son más efectivas y algunos otros han evaluado cuánto más está dispuesto a pagar el consumidor por un producto sustentable o relacionado a una causa. Sin embargo, estos estudios sólo presentan un solo estímulo al participante. La presente investigación evalúa la preferencia del consumidor considerando los tres atributos (i.e., marca, precio, y campaña de RSE) simultáneamente a través de un análisis conjunto. El racional detrás del análisis conjunto es que las personas toman decisiones basándose en un conjunto de elementos y no de uno solo (Lusk y Shogren, 2007). De acuerdo con estos autores una de las ventajas que brinda esta técnica es que no resalta ninguno de los atributos de manera especial, de tal forma que el participante elige su opción preferida en base a todos los estímulos presentados.

\section{El impacto de las campañas de RSE sobre la marca}

La preferencia de una marca por parte del consumidor es una respuesta a los estímulos que recibe filtrados por sus condicionales previos, estos es, generalmente percibe lo que necesita, desea o está de acuerdo a sus intereses (León, 2008). Schiffman y Kanuk (2005) señalan que los consumidores hacen juicios sobre la calidad de un producto considerando atributos intrínsecos del mismo (e.g., color, tamaño, forma, sabor, aroma) y extrínsecos como el precio, la imagen de la marca o reputación del fabricante o distribuidor. 
La teoría de la integración de información apoya esta afirmación pues establece que las actitudes previas del consumidor se integran con la nueva información adquirida para emitir una evaluación hacia la causa, la marca y la relación entre ellas (Lafferty y Goldsmith, 2005).

Entre los atributos extrínsecos, toman relevancia cuatro factores que ayudan a posicionar a una empresa y sus marcas: reputación, responsabilidad social, ética y transparencia (Folse, Niedrich, y Grau, 2010; Krishna y Rajan, 2009; León, 2008). De esta manera, la asociación de una marca con una campaña de RSE tiene un efecto positivo en la marca si el consumidor percibe una acción social genuina por parte de la empresa (Habel, Schons, Alavi y Wieseke, 2016). Por ejemplo, un estudio realizado por la empresa Cone y la Universidad de Duke (2008) muestra que el asociar un producto con una campaña de RSE impacta positivamente a sus ventas. La idea es que la empresa utilice la RSE como un diferenciador de la competencia y que lo conecte con el consumidor. Estudios previos han determinado que el uso de RSE impacta positivamente a empresas con y sin fines de lucro (Baghi y Gabrielli, 2012).

Sin embargo, algunos estudios como el de Baghi, Rubaltelli y Tedeshi (2010) reportan beneficios parciales. De acuerdo a estos autores la utilización de campañas de RSE sólo mejora la imagen de productos hedónicos (i.e., aquellos que se consumen por placer) y no la de los utilitarios. Estos resultados concuerdan con los de Chang (2008) quien defiende que la mercadotecnia con causa es más efectiva con productos frívolos que con los prácticos. Nuestro racional se opone a esta idea y va más en el sentido de la investigación de Gonzáles Hernández, Orozco Gómez y de la Paz Barrios (2011), quienes proponen que la evaluación de los productos se ve influido por las condiciones de involucramiento del consumidor. Para González Hernández et al. (2011) el consumidor realiza una valoración más compleja al decidir la compra de un producto de mayor involucramiento, es decir, aquellos que tienen un valor simbólico y un riesgo percibido más alto. Para la valoración de este tipo de productos se consideran los atributos tangibles pero también los intangibles, como los valores y la imagen de la empresa y sus marcas.

Siguiendo este racional, nosotros proponemos que en productos de alto involucramiento como la leche, la marca constituye un símbolo de calidad y confianza. El consumidor buscará apoyar una marca que resuelve sus necesidades pero que también represente sus valores (Gonzáles Hernández et al., 2011). Las marcas que hacen un mayor esfuerzo de comunicación tienen un mayor reconocimiento del consumidor, es decir, el consumidor está más familiarizado con las marcas que hacen más publicidad. Es más fácil, que la mayoría de los consumidores conozcan los valores de una empresa que se vende a nivel nacional que una que sólo es conocida en una región. El conocimiento de los valores de la empresa le permite al consumidor evaluar si la campaña social emprendida es genuina y merece ser apoyada (Habel, et al., 2016). Por esa razón, y contradiciendo el estudio previo de Arora y Henderson (2007) quienes argumentan que los efectos de la mercadotecnia con causa es mayor para las marcas poco conocidas, proponemos que las campañas de RSE de marcas más reconocidas tendrán una mejor aceptación del consumidor comparada con las de marcas menos conocidas.

Así, la presente investigación propone que existe un efecto directo positivo mayor de las campañas de RSE sobre la preferencia del consumidor en marcas de productos de alto involucramiento (expresado en reconocimiento de marca) comparado con las marcas de medio o bajo involucramiento.

De igual forma, las diferentes campañas de RSE tienen diferente grado de aceptación del consumidor y por lo tanto afecta su efectividad. El estudio de Cone y la Universidad de Duke 
(2008) también muestra que las causas más atractivas para los consumidores son las relacionadas con la salud/enfermedades, el ambiente, y el desarrollo económico de las comunidades. Algunos estudios han evaluado las variables que afectan esta preferencia y la efectividad de la campaña. Por ejemplo, a mayor afinidad entre la causa social y la compañía o el producto el consumidor está dispuesto a donar más (Kuo, y Hamilton, 2015; Arora y Henderson, 2007; Lichtenstein, Drumwright, y Braig, 2004; Pracejus, Olsen, y Brown, 2003). Si el consumidor tiene una percepción favorable de la alianza mejora la percepción tanto de la empresa como de la causa (Habel, et al., 2016; Lafferty, Goldsmith, y Hult, 2004) y la libertad de elegir la causa social incrementa la intención de compra (Robinson, Irmak, y Jayachandran, 2012)

En esta investigación proponemos el siguiente modelo:

Figura 1. Modelo esquematizado propuesto

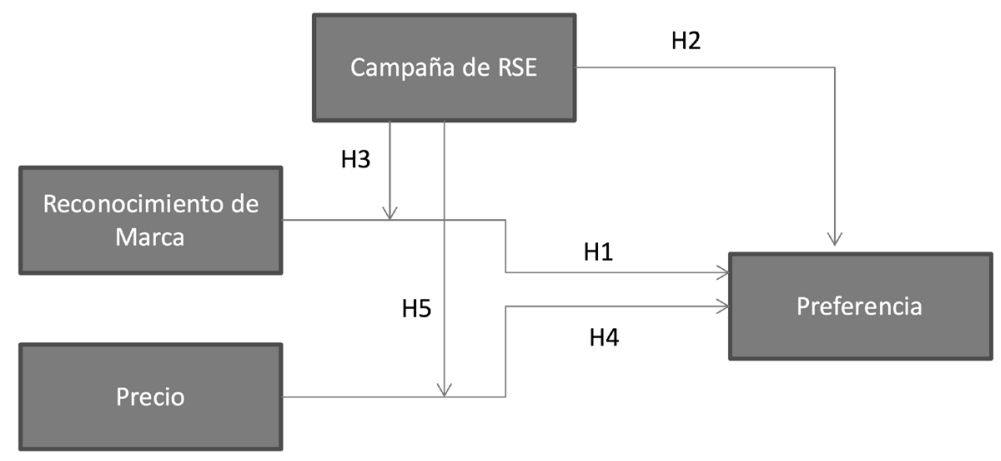

Derivado de lo anterior se presentan las siguientes hipótesis:

H1: En ausencia de campañas de RSE, la preferencia de los consumidores es mayor mientras más reconocida sea la marca.

H2: Una campaña de RSE, ya sea de Causa Social o Ecológica, incrementa la preferencia de los consumidores para todas las marcas.

H3: Una campaña de RSE, ya sea de Causa Social o Ecológica, incrementa la preferencia de los consumidores más en las marcas más reconocidas que en las menos reconocidas.

\section{El efecto de las campañas de RSE sobre el precio}

Las campañas de RSE buscan apoyar a la sociedad pero de igual forma se espera que la compañía/marca patrocinadora reciba algún beneficio. De acuerdo con Edelman (2014) cerca del $80 \%$ de los consumidores en países emergentes no tienen ningún problema en aceptar que una empresa o marca haga una campaña de responsabilidad social y a la vez obtenga cierta utilidad. Dichos consumidores están dispuestos a confiar más y a recomendar a las marcas que apoyan una causa social, incluso si implica cambiar de marca. De igual forma, el estudio reporta que el $54 \%$ de los consumidores están dispuestos a pagar un sobreprecio por productos de dichas marcas. 
Tradicionalmente la teoría económica argumenta que las personas buscan racionalmente maximizar la utilidad (Simon, 1959). Bajo esta teoría el precio que se paga por un producto es un costo que se debe limitar. El consumidor busca comprar los mejores productos al mejor precio posible. Así desde la perspectiva del consumidor el precio tiene una imagen negativa.

Sin embargo, basándonos en los estudios de opinión como el de Eldeman y en base a estudios académicos previos que reportan una intención de pagar más por productos producidos de una forma socialmente responsable (Alphonce y Alfnes, 2012; Ha-Brookshire y Norum, 2011; Subrahmanyan, 2004) proponemos que las campañas de RSE atenúan esta percepción negativa.

Algunos otros estudios refuerzan esta idea. Henderson y Arora (2010) encontraron que la mercadotecnia con causa es más efectiva que una reducción en el precio. KoschateFischer, Stefan y Hoyer (2012) y Popkowski-Leszcczyc y Wong (2010) argumentan que a medida que aumenta el monto de la compra donado a la causa se incrementa el precio que los consumidores están dispuestos a pagar por el producto. Las solicitudes de cantidades cerradas (precios o donativos en números absolutos) son más efectivas que las expresadas en porcentajes, por la facilidad para entenderlas (Chladek, Florack y Kleber, 2013). De igual forma, Winterich y Barone (2011) concluyen que las personas con un perfil interdependiente (menos independiente) prefieren adquirir productos asociados a una causa que con descuento en precio. Más recientemente, Habel et al (2016) descubrieron que los consumidores están dispuestos a pagar más por un producto socialmente responsable (i.e. lo consideran justo) si confían en la empresa y consideran que el aumento de precio no es sólo una estrategia financiera para aumentar sus utilidades.

Como lo muestra la figura 1, en este estudio proponemos las siguientes hipótesis referentes a la disposición de los consumidores a pagar un sobreprecio:

\section{H4: En ausencia de campañas de RSE, incrementos en el precio del producto disminuyen la preferencia de los consumidores \\ H5: Una campaña de RSE, ya sea de Causa Social o Ecológica, disminuye el efecto negativo del precio en la preferencia de los consumidores}

Las cinco hipótesis del presente trabajo se pueden describir en la Figura 1. Los efectos directos o marginales del reconocimiento de marca (H1), la campaña de RSE (H2), y del precio (H4) se describen en los paths o flechas que van de estas tres variables a las preferencias. El modelo incluye también dos efectos indirectos por los cuales puede operar las campañas de RSE, interactuando con el reconocimiento de la marca (H3) o disminuyendo el castigo en las preferencias que ocasiona un aumento de precios (H5). Estos efectos indirectos se denominan en la literatura como "moderadores" (Hayes 2013), ya que implican que el efecto de una variable independiente (i.e. marca o precio) sobre la variable dependiente (i.e. preferencia), es distinta si está presente o no otra variable, en nuestro caso la campaña de RSE, y se representan por las flechas que salen de RSE a los paths de reconocimiento de marca y de precio con las preferencias.

En este sentido la RSE modera el efecto entre la marca y las preferencias y el precio y las preferencias lo que lo hace convertirse en una variable moderadora. A esta variable también se le llama interacción, ya que el efecto de la marca y/o el precio ya no es independiente de la RSE sino que interactúa con él. En el resto del escrito tomaremos como sinónimos los términos de interacción y moderación. 


\section{Metodología}

Para la aplicación del análisis conjunto en este estudio elegimos tres atributos (1) un producto de uso frecuente, básico y de alto involucramiento, (2) diferentes campañas de responsabilidad empresarial, y (3) distintos niveles de precio para poder armar diversos ofrecimientos de venta. Se eligió la leche porque es un producto conocido y adquirido por una gran proporción de la población adulta. De acuerdo con la Organización de las Naciones Unidas para la Alimentación y la Agricultura más de 6,000 millones de personas consumen leche en el mundo. De acuerdo a esta organización la mayor parte de América Latina, incluyendo México, tienen un consumo de leche que va de los 30 a los 150 kilogramos per cápita por año (FAO, 2015). De hecho el consumo de leche per cápita va en aumento como consecuencia de la urbanización, el cambio en los hábitos alimenticios, y en el crecimiento de los ingresos. La leche es también un producto que requiere un mayor involucramiento pues se relaciona con la salud y bienestar de la familia (FAO, 2015).

Una vez elegido el producto, hicimos un estudio por medio de redes sociales para identificar las marcas más conocidas y de mayor confianza en el mercado mexicano y cuáles son las marcas conocidas sólo en determinadas regiones. Las respuestas obtenidas señalan a la marca Lala como la marca más conocida en México, Nutri Leche como una marca medianamente reconocida y la marca Carranco como una marca poco conocida. De esta forma obtuvimos tres marcas cuyo nivel de reconocimiento va de alto a bajo.

Para el atributo de la campaña de RSE buscamos en reportes de encuestas realizadas a nivel mundial las causas que más importan a los consumidores. De acuerdo con Edelman (2012) el $89 \%$ de los consumidores entrevistados a nivel mundial eligieron las campañas que mejoran la salud de las personas y las que protegen al medio ambiente como las que más les gustaría apoyar. Para nuestro estudio, decidimos agregar una tercera campaña que hiciera la función de una campaña neutral y que sirviera como referencia para las otras dos opciones. Elegimos la campaña de apoyo a los derechos de los animales (i.e., evitar el maltrato de los animales en las granjas productivas), por considerarla una campaña que está alineada al negocio de la industria láctea y que estudios previos como el de Gupta y Pirsch (2006) argumentan que incrementa las intenciones de compra.

Por último, el estudio de Goodpurpose (Edelman, 2014) reporta que el $54 \%$ de los consumidores estan dispuestos a pagar un sobreprecio por productos de marcas asociadas a una causa social. Así que para nuestro tercer atributo decidimos elegir el precio de mercado de un litro de leche en envase tetrapack (i.e., \$15.50) y definimos dos niveles de sobreprecio. Se establecieron un precio moderadamente alto de $\$ 19.00$ pesos con un $22.5 \%$ de incremento y un precio alto de $\$ 22.00$ pesos con un $41.9 \%$ de incremento. Estos sobreprecios están basados en estudios anteriores que demuestran que los consumidores están dispuestos a pagar hasta un $40 \%$ de sobreprecio por productos socialmente responsables y asociados a una causa (e.g., Amezcua, 2015; Alphonce y Alfnes, 2012; Ha-Brookshire y Norum, 2011; Subrahmanyan, 2004).

De esta forma el análisis conjunto en este estudio se realizó considerando 3 atributos con tres niveles cada uno, lo que nos da 27 combinaciones de atributos diferentes. Se elaboró una tarjeta con cada una de las combinaciones para que los participantes las ordenaran de acuerdo a su preferencia. 
El estudio se diseñó para ser administrado vía electrónica y así lograr una cobertura nacional y con mayor diversidad de participantes que tengan acceso a internet o a dispositivos móviles inteligentes. La posibilidad de participar en el estudio desde cualquier dispositivo móvil con conexión a internet permitió optimizar los recursos económicos y de tiempo. Sin embargo, las dimensiones reducidas de los dispositivos móviles dificultaban la revisión y ordenamiento de las 27 tarjetas, así que optamos por reducir el número de tarjetas a través de los diseños ortogonales de Taguchi que han demostrado su efectividad en estudios previos de calidad y desarrollo de nuevos productos (e.g., Raajpoot, Javed, y Koh, 2008). Los resultados obtenidos con los diseños factoriales completos y con los arreglos fraccionados de Taguchi son muy similares. Las tarjetas que fueron seleccionadas siguiendo la metodología de Taguchi y que se utilizaron en el presente estudio se muestran en el Anexo 1. A cada participante se le pidió que ordenara las 9 tarjetas en base a su preferencia y posteriormente se le aplicaba una batería de reactivos para establecer sus demográficos y sus patrones de consumo de leche. El instrumento electrónico se distribuyó a través de e-mail y de las redes sociales entre los meses de julio y agosto 2015 obteniéndose 2043 observaciones.

Es claro que al usar medio electrónicos los resultados pueden no ser representativos del universo nacional. Esta es una de las limitantes del estudio, misma que abordamos en las conclusiones donde sugerimos otros medios para reforzar la representatividad. Aun así, las redes sociales han resultado útiles en investigaciones sociales porque en ella los participantes gustan de interactuar y participar voluntariamente en dinámicas como pruebas de personalidad y, como en nuestro caso, en encuestas (Ellison, Steinfeld, y Lampe, 2007). Aunado a esto, investigaciones han reportado que no hay diferencias significativas en utilizar métodos de recolección en redes sociales con su contraparte presencial (Birnbaum, 2004; Grieve, Witteveen y Tolan, 2014). Para el caso mexicano, la Asociación Mexicana de Internet (AMIPCI) reporta que al 2015 el 53\% de la población mexicana utiliza regularmente el Internet. El 76\% de los usuarios de internet en México tienen entre 13 y 44 años. El 46\% de ellos pertenece al nivel socioeconómico $\mathrm{C} / \mathrm{D}+\mathrm{y}$ el $47 \%$ a las clases superiores $(\mathrm{C}+\mathrm{y} \mathrm{A} / \mathrm{B})$ con una proporción igual entre hombres y mujeres. En base a estas cifras, la AMIPCI ratifica como aceptable el uso de encuestas electrónicas para la realización de investigación de mercados. De hecho, la AMIPCI encontró que más de la mitad de la población en México son internautas habituales, con una tasa de crecimiento anual de hasta $13 \%$ en los últimos dos años. Estas estadísticas van de acuerdo a las cifras globales donde, por ejemplo, los países europeos observan un crecimiento del $11 \%$ anual y la población con acceso internet ronda el $50 \%$ de la población (We are social, 2015).

En el caso de las redes sociales, éstas han tomado un papel preponderante en los últimos años. En México, el 89\% de los usuarios de Internet hacen uso de estas redes (AMIPCI, 2015). México sigue la tendencia mundial del uso de las redes sociales, mostrando un crecimiento del 12\% anual y con Facebook como líder en el ramo, con más de1.366 millones de usuarios reportados en enero del 2015 (We are social, 2015).

\section{Modelo de investigación propuesto}

La campaña de RSE puede afectar de forma directa a las preferencias o de una forma indirecta a través de una interacción con alguna otra variable o de ambas formas. En particular proponemos que la campaña de RSE incrementa positivamente las preferencias, pero también 
puede hacer la calificación de preferencia más insensible a los precios, es decir un precio más alto castigaría la preferencia menos si hay campaña de RSE a si no la hay, o puede hacer más atractiva a la marca líder en comparación a la menos posicionada (ver figura 1). Así, podemos utilizar un modelo de preferencia lineal del tipo

$$
\begin{aligned}
& U_{i t}=\alpha+\gamma_{s} S_{i t}+\gamma_{e} E_{i t}+\beta_{p} P_{i t}+\beta_{s}\left(P_{i t} * S_{i t}\right)+\beta_{e}\left(P_{i t} * E_{i t}\right)+\delta_{C} C_{i t}+\delta_{m} M_{i t}+ \\
& \pi_{s}\left(C_{i t} * S_{i t}\right)+\pi_{e}\left(C_{i t} * E_{i t}\right)+\mu_{c}\left(M_{i t} * S_{i t}\right)+\mu_{e}\left(M_{i t} * E_{i t}\right)+\varepsilon_{i t}
\end{aligned}
$$

Donde $U$ denota preferencia que va del 1 al 9, donde 1 es el menos preferido y 9 el más preferido, $S$ es una variable dicotómica que toma el valor 1 si la campaña de RSE es de causa social y 0 si no, $E$ toma el valor 1 si la campaña de RSE es de índole ecológica y 0 si no, $P$ es el precio de cada alternativa expresada en pesos por unidad, $C$ toma el valor 1 si la marca es conocida y 0 si no y finalmente $M$ es una dicotómica que toma el valor de 1 si la marca es medianamente conocida y 0 si no. Los subíndices i y t se refieren a los individuos y a las tarjetas que evaluaron, los coeficientes $\alpha$, $\gamma$ 's, $\beta$ 's, $\delta$ 's, $\pi$ 's y $\mu$ 's son los parámetros del modelo y $\varepsilon$ son los residuales.

Así, los efectos de armar una campaña de RSE ya sea de causa social o ecológica, para la marca más reconocida se pueden obtener como:

$$
\Delta U_{i t}=\gamma_{j}+\beta_{j} P_{i t}+\pi_{j}
$$

Y es igual a (3) para la marca medianamente reconocida:

$$
\Delta U_{i t}=\gamma_{j}+\beta_{j} P_{i t}+\mu_{j}
$$

Donde “j”" puede denotar la campaña de causa social o la ecológica. Como se puede observar en la ecuación (2) y (3), el efecto directo está dado por $\gamma_{\mathrm{j}}$, mientras que el que opera a través de los precios por $\beta_{\mathrm{j}}$ y el que opera a través del reconocimiento de la marca por $\pi_{\mathrm{j}}$ y $\mu_{\mathrm{j}}$.

Adicionalmente, podemos expresar el incremento en la preferencia o utilidad en pesos de una campaña de RSE de la siguiente forma. Primero estimamos el incremento en las preferencias producto de la campaña de RSE tanto por sus efectos directos como los indirectos a través de la moderación en los precios y el reconocimiento de marca. El segundo paso es estimar cuánto tendría que reducir el precio para obtener el mismo incremento en la preferencia que otorgaría la campaña de RSE “j” y que se estimó en el primer paso, si ésta no se implementara. A esta reducción de precios le llamaremos el descuento equivalente y el descuento equivalente lo podemos poner en proporción al precio para obtener el precio relativo, mismo que denominaremos como el premio equivalente de la campaña de RSE. Esto denota el premio en el precio que permite mantener la campaña de RSE, dado que en su ausencia, se tendría que bajar el precio para obtener el incremento en la preferencia y el premio se evaporaría.

Para estimar el descuento equivalente para la marca más reconocida simplemente igualamos el incremento de la preferencia dado por (2) al incremento en la utilidad si reducimos el precio. En símbolos se representa con la ecuación:

$$
\Delta U_{i t}=\gamma_{j}+\beta_{j} P_{i t}+\pi_{j}=\beta_{p} \Delta P_{i t}
$$


Despejando para el cambio en precios:

$$
\Delta P_{i t}=\frac{\gamma_{j}+\pi_{j}+\beta_{j} P_{i t}}{\beta_{p}}
$$

El premio equivalente se puede estimar simplemente como:

$$
\text { Premio }=-\frac{\Delta P_{i t}}{P_{i t}}=-\frac{\gamma_{j}+\pi_{j}+\beta_{j} P_{i t}}{\beta_{p} P_{i t}}
$$

el cual se multiplica por el signo menos para expresarlo como un sobre precio, ya que el descuento equivalente necesariamente implica una reducción de precio, por lo que es negativo.

De igual forma, para la marca medianamente reconocida el premio equivalente es igual a:

$$
\text { Premio }=-\frac{\Delta P_{i t}}{P_{i t}}=-\frac{\gamma_{j}+\mu_{j}+\beta_{j} P_{i t}}{\beta_{p} P_{i t}}
$$

Entonces para estimar los efectos directos e indirectos, y su valuación en pesos mediante el descuento y el premio en precio equivalente, lo que requerimos es la estimación de los parámetros del modelo de la ecuación (1). La estimación puede realizarse con el método de mínimos cuadrados ordinarios (pooling) debido a que es un estudio donde las variables precio, campaña social y grado de conocimiento de la marca fueron manipulados ortogonalmente por los investigadores. Esto permite que los residuales no estén correlacionados con los regresores. Asimismo, dado que cada individuo evalúa varias repeticiones de cada atributo, podría estimarse con métodos de datos panel ya sea de efectos fijos o de efectos aleatorios. Sin embargo, como las alternativas o tarjetas son las mismas para cada individuo, entonces la varianza entre individuos es cero, por lo que los tres modelos (pooling, efectos fijos y aleatorios) arrojan las mismas estimaciones (Wooldridge, 2009).

\section{Análisis de Resultados}

En términos de las personas que participaron en este estudio, la tabla 1 muestra que el $97 \%$ de los entrevistados tienen entre 18 y 49 años. Siendo los de 21 a 29 años el grupo de edad más numeroso con un $40 \%$. La muestra estuvo equilibrada entre hombres y mujeres. Estos resultados coinciden con los resultados previos de la AMIPCI (2015), por lo que se considera que la muestra en este estudio representa al sector de la población mexicana que tiene acceso a internet y redes sociales. Aunado a esto, del total de los encuestados, el $73 \%$ son solteros y el $51 \%$ tiene un trabajo. De igual forma el estudio tuvo una participación del $41.5 \%$ de gente que trabaja y un $41 \%$ de estudiantes. La escolaridad de los participantes fue variada pero los porcentajes más fuertes son preparatoria $(27.75 \%)$, universidad (48\%) y posgrado (22\%). Aunque el nivel socioeconómico no se determinó a través de preguntas (e.g. niveles socioeconómicos AMAI), la mayoría de las encuestas se realizaron a través de los contactos de estudiantes en universidades públicas, consideramos que cubre las clases D+ (Bajo/alto), C (Medio) y C+ (Medio/alto) por lo que se considera que los datos obtenidos son válidos para dichos niveles socioeconómicos y no para los niveles D (Bajos ingresos) y E (Pobreza extrema). 
Tabla 1

Demográficos de los entrevistados

\begin{tabular}{|c|c|c|c|}
\hline Categoría & Rango/Valor & Frecuencia & $\%$ \\
\hline \multirow[t]{8}{*}{ Edad } & 17 o menos & 2 & 0.88 \\
\hline & $18-20$ & 46 & 20.26 \\
\hline & $21-29$ & 93 & 40.97 \\
\hline & $30-39$ & 57 & 25.11 \\
\hline & $40-49$ & 25 & 11.01 \\
\hline & $50-59$ & 3 & 1.32 \\
\hline & Más de 60 & 1 & 0.44 \\
\hline & & 227 & 100 \\
\hline \multirow[t]{3}{*}{ Género } & Masculino & 98 & 43.17 \\
\hline & Femenino & 129 & 56.83 \\
\hline & & 227 & 100 \\
\hline \multirow[t]{6}{*}{ Edo Civil } & Soltero & 166 & 73.13 \\
\hline & Casado & 55 & 24.23 \\
\hline & Separado & 2 & 0.88 \\
\hline & Divorciado & 3 & 1.32 \\
\hline & Viudo & 1 & 0.44 \\
\hline & & 227 & 100 \\
\hline \multirow[t]{6}{*}{ Ocupación } & Desempleado & 14 & 6.17 \\
\hline & Medio Tiempo & 25 & 11.01 \\
\hline & Tiempo Completo & 92 & 40.53 \\
\hline & Estudiante & 94 & 41.41 \\
\hline & Retirado & 2 & 0.88 \\
\hline & & 227 & 100 \\
\hline \multirow[t]{5}{*}{ Escolaridad } & Universidad & 110 & 48.46 \\
\hline & Secundaria & 3 & 1.32 \\
\hline & Posgrado & 51 & 22.47 \\
\hline & Preparatoria & 63 & 27.75 \\
\hline & & 227 & 100 \\
\hline
\end{tabular}

Para efectos de probar las hipótesis establecidas previamente se construyeron cuatro modelos los cuales pueden observarse en la Tabla 2. En estos cuatro modelos se incluyeron los predictores marca, precio, campaña social y campaña ecológica. El primer modelo considera solamente los cuatro predictores sin interacciones, es decir asume que el efecto de las campañas sociales sobre la preferencia es solo el directo. Los modelos (2) al (4) incluyen interacciones, o permiten introducir el efecto de la campaña social sobre el precio. 
Tabla 2. Modelos de regresión pooling

\begin{tabular}{|c|c|c|c|c|}
\hline & (1) & (2) & (3) & (4) \\
\hline \multirow[t]{2}{*}{ Constante } & $7.904165 * * *$ & $7.832414 * * *$ & $7.739689 * * *$ & $7.739689 * * *$ \\
\hline & $(0.3795224)$ & $(0.5705921)$ & $(0.4809433)$ & $(0.4809433)$ \\
\hline \multirow[t]{2}{*}{ Marca Conocida (C) } & $2.223201 * * *$ & $2.394889^{* * *}$ & $2.214315^{* * *}$ & $2.214315^{* * *}$ \\
\hline & (0.1250437) & (0.1602393) & 0.1767066 & $(0.1767066)$ \\
\hline \multirow{2}{*}{$\begin{array}{l}\text { Marca Medianamente } \\
\text { Conocida (M) }\end{array}$} & $0.3582966^{* * *}$ & $0.354825^{* *}$ & $0.5109785^{* * *}$ & $0.5109785^{* * *}$ \\
\hline & $(0.1250437)$ & $(0.176659)$ & 0.158401 & $(0.158401)$ \\
\hline \multirow[t]{2}{*}{ Campaña Ecológica (E) } & -0.135 & & & \\
\hline & $(0.1250437)$ & & & \\
\hline \multirow[t]{2}{*}{ Campaña Causa Social (S) } & $0.6020558 * * *$ & $0.6020558 * * *$ & & 0.903624 \\
\hline & $(0.1250437)$ & $(0.1249475)$ & & (1.091518) \\
\hline \multirow[t]{2}{*}{ Precio (P) } & $-0.2081585 * * *$ & $-0.207326 * * *$ & $-0.2055569 * * *$ & $-0.2055569 * * *$ \\
\hline & $(0.0192185)$ & $(0.0271582)$ & $(0.0271655)$ & 0.0271655 \\
\hline \multicolumn{5}{|l|}{$\begin{array}{l}\text { Interacciones de las } \\
\text { Campañas RSE con Marca }\end{array}$} \\
\hline \multirow{2}{*}{$\begin{array}{l}\text { Marca Conocida*Ecológica } \\
\left(C^{*} \mathrm{E}\right)\end{array}$} & & -0.5225418 & & \\
\hline & & $(0.3436865)$ & & \\
\hline \multirow{2}{*}{$\begin{array}{l}\text { Marca Medianamente } \\
\text { Conocida*Ecológica }(M * E)\end{array}$} & & -0.0034744 & & \\
\hline & & $(0.4583593)$ & & \\
\hline \multirow{2}{*}{$\begin{array}{l}\text { Marca Conocida*Causa } \\
\text { Social }(\mathbf{C} * \mathbf{S})\end{array}$} & & & 0.2669798 & \\
\hline & & & $(0.3224939)$ & \\
\hline \multirow{2}{*}{$\begin{array}{l}\text { Marca Medianamente } \\
\text { Conocida*Causa Social } \\
(\mathbf{M} \text { S })\end{array}$} & & & -0.3471276 & $-0.470349 * *$ \\
\hline & & & $(0.2689073)$ & $(0.2298314)$ \\
\hline \multicolumn{5}{|l|}{$\begin{array}{l}\text { Interacciones de las } \\
\text { Campañas de RSE con } \\
\text { Precio }\end{array}$} \\
\hline \multirow[t]{2}{*}{ Precio*Ecológica $(\mathbf{P} * \mathbf{E})$} & & 0.0021368 & & \\
\hline & & $(0.0154662)$ & & \\
\hline \multirow[t]{2}{*}{ Precio*Causa Social $(\mathbf{P} * \mathbf{S})$} & & & $0.0369727 * * *$ & -0.0041011 \\
\hline & & & $(0.0103449)$ & $(0.0576552)$ \\
\hline \multicolumn{5}{|l|}{$\begin{array}{l}\text { F (p value) de Prueba de } \\
\text { Wald (F) de Ho }\end{array}$} \\
\hline $\begin{array}{l}\text { Campaña Ecológica no tiene } \\
\text { impacto }\end{array}$ & & $2.1(0.098)$ & & \\
\hline
\end{tabular}




$\begin{array}{lllll}\begin{array}{l}\text { Suma de Errores al } \\ \text { Cuadrado }\end{array} & 10845.0535 & 10817.7465 & 10828.8948 & 10828.8948 \\ \text { R2 ajustada } & 0.2018 & 0.203 & 0.2026 & 0.2026 \\ \mathbf{F} & 104.24 & 75.31 & 87.46 & 87.46 \\ * * * \mathbf{p}<\mathbf{0 . 0 1}, * * \mathbf{p}<\mathbf{0 . 0 5} & & & & \end{array}$

Los coeficientes de las variables consideradas en el modelo 1 son significativos $(\mathrm{p}<.01)$ y son los esperados con excepción del coeficiente de la campaña ecológica en la regresión 1. Así por ejemplo la marca más conocida incrementa la preferencia en 2.2 unidades $(\beta 1)$ o $44 \%(2.2 / 5)$ sobre la media (5) en la escala de preferencia del 1 al 9, mientras que la marca medianamente conocida solo incrementa la preferencia en 0.36 unidades $(\beta 2)$ o $7 \%(0.36 / 5)$. El coeficiente del precio $(\beta 3)$ indica que por cada peso que aumenta el precio, la preferencia cae en 0.21 unidades. Esto significa una elasticidad de la preferencia a los precios de -0.8 calculada en el precio y preferencia mediana $(0.21 \times \$ 18.33 / 5)$. Por otra parte, incluir una campaña de causa social incrementa en forma directa la preferencia en 0.602 unidades $(\beta 4)$ o $12 \%(0.602 / 5)$, en comparación a no incluirla. En contraste, incluir una campaña ecológica no tiene efecto alguno para este modelo. La bondad de ajuste muestra que la marca, el precio y el tipo de campaña explican una quinta parte de las variaciones en la preferencia.

La ecuación 2 muestra con más detalle si la campaña ecológica tiene algún efecto moderador tanto en el efecto del reconocimiento de marca en las preferencias como en el de los precios en las preferencias. El punto a destacar de esta estimación es que nuevamente no es posible rechazar el que la campaña ecológica tenga efecto alguno, directo o indirecto, sobre las preferencias. De hecho, la prueba de Wald muestra que todos los coeficientes donde interviene la campaña de RSE de naturaleza ecológica son cero y no puede rechazarse al 5\%. Este resultado significa que incluir en este caso una campaña de RSE de tipo ecológica o no incluirla da lo mismo. Claramente, este resultado sólo es aplicable en este caso y no puede extrapolarse esta conclusión a las diferentes campañas de índole Ecológica ni a todas las categorías de productos disponibles.

Por este motivo, y dado que la campaña ecológica resultó no significativa corrimos el modelo (3) eliminando esta variable y sus interacciones dejando solamente la campaña de RSE de causa social. En la ecuación (3) incluimos todas las interacciones posibles, pero no el efecto marginal o directo de la campaña de RSE de causa social. Al parecer los coeficientes de las interacciones de la campaña con el reconocimiento de la marca no son significativas al 5\%. En la ecuación (4) ensayamos incluir solo la interacción más prometedora, que es con la marca medianamente reconocida, y resultó significativa al 5\%.

La ecuación (4) es la ecuación que captura mejor los efectos de la campaña de RSE de causa social sobre las preferencias. Los coeficientes positivos, significativos y mayores para la marca reconocida que para la medianamente reconocida, apoyan la $\mathrm{H} 1$ es decir mientras mayor el reconocimiento de la marca mayor las preferencias. El coeficiente negativo y significativo del precio es consistente con $\mathrm{H} 4$, esto es, al incrementarse el precio se reducen las preferencias. 
El coeficiente negativo de la interacción de la campaña de RSE con la variable dicotómica de marca medianamente reconocida del orden de -0.47 indica que el efecto de la campaña de RSE va a ser mayor para la marca más reconocida que para la medianamente reconocida, lo que apoya la $\mathrm{H} 3$.

Ahora bien, los coeficientes de la campaña de RSE de causa social y la interacción de la campaña con el precio aparecen como no significativos distintos de cero. Sin embargo, en este punto se debe hacer una aclaración importante, cuando la variable de la campaña pasa de 0 a 1 , se dan diferentes efectos, el directo y los que operan a través de la marca medianamente reconocida y el de los precios. En cambio, el estadístico " t" está diseñado para ver el efecto de una variable sobre la dependiente manteniendo el resto fijas, lo que claramente no es el caso. Por esta razón, el estadístico de significancia es el de Wald, específicamente la prueba de la hipótesis nula de que todos los coeficientes donde aparece la variable de la campaña de RSE con causa social son cero. El estadístico se presenta en la columna (4) y arroja una $\mathrm{F}=14.16$ que es significativamente distinta de cero al 1\%. De hecho, el estadístico de Wald para la hipótesis nula donde los coeficientes de la campaña de causa social y el de la interacción del precio sean cero se rechaza también al 1\% $(\mathrm{F}=19.44)$

Como mencionamos los estadísticos " $\mathrm{t}$ " no son particularmente útiles cuando una variable, como la campaña de RSE de causa social, aparece en varias variables al mismo tiempo. Adicionalmente, precisamente porque las interacciones se construyen a partir de la multiplicación de esta variable con el resto de las independientes, se introduce colinealidad entre las variables, lo que tiende a inflar las varianzas y disminuir los estadísticos " $t$ ". De hecho este es el caso, el factor de inflación de la varianza (VIF por sus siglas en inglés), que mide cuantas veces es mayor la varianza de los coeficientes en comparación al caso de cero colinealidad, es de 103 para la interacción de la campaña de RSE con el precio y de 101 para la variable del RSE. También por esta razón no debe de tomarse el estadístico "t” como la prueba de significancia sino más bien las pruebas de Wald que involucran todos los coeficientes donde aparece la variable de la campaña de $\mathrm{RSE}^{5}$.

Una vez obtenidos los coeficientes en la regresión, se puede estimar los descuentos y premios equivalentes utilizando las ecuaciones (5), (6) y (7) para la especificación final del modelo que es la ecuación (4). En el caso de las estimaciones del modelo (4), El incremento total en la preferencia de la campaña de RSE con causa social evaluado en la media de los precios (18.33) para la marca más reconocida es igual a $0.826=0.904-0.004 * 18.33$, mientras el incremento en la preferencia de la marca medianamente reconocida es igual a $0.358=0.904-0.470-0.004 * 18.33$. De esta forma, para obtener estos incrementos en las preferencias el descuento equivalente en los precios debería de ser igual a 4.020 pesos para la marca más reconocida y 1.742 para la medianamente reconocida, lo que equivale a un premio de la campaña de RSE con causa social de casi $22 \%$ para la marca más reconocida y de $10 \%$ para la medianamente reconocida.

\footnotetext{
${ }^{5}$ Una alternativa para lidiar con la multicolinealidad es emplear el método de Ridge Regression, que contrae los estadísticos a estimar para reducir la inflación en las varianzas, pero al costo de introducir sesgos. Empleando este método los resultados no difieren sustancialmente de las estimaciones reportadas. Las campañas con causas incrementan la preferencia en las marcas reconocidas en 0.8 y en las medianamente reconocidas en 0.4 en las estimaciones originales, mientras que con las del método Ridge se incrementan las preferencias en 0.77 y 0.39 respectivamente. Por tal motivo dejamos las estimaciones originales.
} 


\section{Discusión y Conclusión}

Los resultados de nuestro estudio nos permiten concluir que las preferencias de los consumidores se incrementan si el producto está vinculado a una campaña de RSE de causa social, más no si la naturaleza de la RSE es Ecológica. Así, al menos para las RSE de causa social, la evidencia del ejercicio de Análisis Conjunto es consistente con las hipótesis H1 a H5. Es decir, las preferencias tienen una relación directa con el reconocimiento, la campaña de causa social impacta directamente a las preferencias, y modera el efecto del reconocimiento de marca y los precios con las preferencias.

No sólo eso, sino que nuestras estimaciones apuntan que, para este caso, la sola inclusión de la causa social incrementa la preferencia entre 0.4 y 0.8 unidades en una escala de 1 a 9 o dependiendo del reconocimiento de la marca, es decir entre 8 y $16 \%$ en relación a la preferencia mediana en comparación a no incluirla. Esto equivale a que la campaña de causa social permite a la empresa gozar de un premio equivalente en el precio de $22 \%$ si la marca es reconocida y solo $10 \%$ si es medianamente reconocida. Esto apoya la $\mathrm{H} 3$ que establece que la campaña de RSE tiene más rentabilidad en la marcas más reconocidas.

El método del descuento o premio del precio equivalente propuesto en este artículo puede aplicarse para estimar el valor total de la campaña. Simplemente habría que multiplicar el premio del precio sobre el volumen de ventas típico que tiene la empresa, o en este caso, 1.7 y 4 pesos por cada litro vendido para cada marca. Esta estimación debe considerar un límite inferior ya que la campaña de RSE podría aumentar el número de unidades vendidas, posibilidad no examinada en este estudio.

El estudio tiene algunas limitaciones que representan potenciales líneas futuras de investigación. Una limitación es que sólo consideramos una categoría de producto, la leche, y los resultados podrían variar en gran medida dependiendo de si el producto asociado a la campaña de RSE es de categoría de lujo o hedónico versus necesario (Koschate-Fischer, Stefan, y Hoyer, 2012) o de categoría saludable en comparación a aquellas que pueden dañar la salud. Por lo tanto, futuras investigaciones podrían considerar no solamente una nueva categoría de producto si no la comparación entre ellas para estimar las variaciones en las elasticidades de precios.

En todo caso, nuestros resultados indican que solo algunas campañas de RSE tienen efectos en el consumidor. En este caso particular orientar la campaña a cuestiones ecológicas no parece tener efectos sobre la preferencia, lo cual puede indicar que los consumidores dan prioridad a ayudar a los más desprotegidos en lugar de cuidar el medio ambiente. Una posible razón por la cual la campaña ecológica no tuvo efecto en este estudio es quizá por el tipo de producto que se utilizó para recolectar los datos. La leche, es un producto de alto involucramiento asociado a otros valores por ejemplo la nutrición o la niñez. Futuras investigaciones pueden comprobar si las campañas ecológicas tienen efecto en otro tipo de productos.

Otra limitación de nuestro estudio es la composición de la muestra, pues el instrumento se distribuyó a través de medios electrónicos. Debido al procedimiento muestral seguido, nuestra muestra representa únicamente al 53\% de la población mexicana (AMIPCI, 2015). Nuestros hallazgos son extrapolables solo a la población que tiene acceso a medios electrónicos, a los niveles socioeconómicos D+ y superiores que tienen niveles de estudio mínimos de secundaria. En una segunda fase de esta investigación se debería considerar la población de bajos recursos económicos (D) y con niveles de educación más bajos, además de considerar distribuir 
el instrumento a través de un medio que permita la representatividad de toda la población, inclusive de forma presencial. También podría considerarse otro tipo de productos; por ejemplo, productos funcionales, de tentación u otros.

Por último, este estudio utilizó marcas de leche en las que confían los consumidores. Sería interesante analizar en una siguiente fase, el impacto en la preferencia o intención de compra de una campaña de RSE, cuando la gente no confía en la marca y se realiza un incremento en el precio de venta.

Aún y con estas limitantes, nuestros resultados implican que las organizaciones deben reconocer la importancia de las campañas de RSE en el momento de diseñar estrategias de mercado. El diseño de estas estrategias requiere que se identifique el tipo de campaña que más impacte las preferencias de los consumidores. Esto permite que a través de un posicionamiento socialmente responsable se logre una diferenciación efectiva y se refuerce la conciencia de las marcas y las empresas en mercados de alta competencia. No sólo se trata de una implicación cualitativa, sino que este estudio también sugiere que la adhesión a campañas de RSE que los consumidores consideran relevantes, pueden incrementar la rentabilidad, en doble dígito, antes de aportar a la causa. Esto demuestra que la RSE "paga" o es rentable desde el punto de vista económico, es decir, la campaña social mitiga el impacto en incremento en precios así como, al mismo tiempo, genera impacto social derivado de la implementación de las campañas.

\section{Referencias}

Aaker, J. \& Keller, K. L. (1990). Consumer evaluations of brand extensions. Journal of Marketing, 54(1), 27-41. https:/ doi.org/10.2307/1252171

Alphonce, R. \& Alfnes, F. (2012). Consumer willingness to pay for food safety in Tanzania an incentive-aligned conjoint analysis. International Journal of Consumer Studies, 36(4), 394-400. https://doi.org/10.1016/j.foodpol.2014.06.009

Amezcua, B. (2015). The Impact of Temporal distance in Willingness to Pay for Cause-Related Products (Tesis doctoral). EGADE Business School, Monterrey, México.

AMIPCI (2015). 11 o estudio sobre los hábitos de los usuarios de internet en México 2015. Accesado el 11 de abril del 2016. Disponible en https://amipci.org.mx/images/AMIPCI_HABITOS_DEL_INTERNAUTA_MEXICANO_2015.pdf

Arora, N. \& Henderson, T. (2007). Embedded Premium Promotion: Why It Works and How to Make It More Effective. Marketing Science, 26(4), 514-531. https://doi.org/10.1287/mksc.1060.0247

Baghi, I. \& Gabrielli, V. (2012). For-profit or non-profit brands: which are more effective in a cause-related marketing programme? Journal of Brand Management, 20, 218-231. https://doi.org/10.1057/bm.2012.35

Baghi, I., Rubaltelli, E. \& Tedeschi, M. (2010) Mental accounting and cause related marketing strategies. International Review of Public and Nonprofit Marketing, 7(2), 145-156. https://doi.org/10.1007/s12208-010-0055-4

Barone, M.J., Miyazaki, A.D. \& Taylor K.A. (2000). The influence of cause related marketing on consumer choice: Does one good turn deserves another? Journal of the Academy of Marketing Science, 28(2), 248-262. https://doi. org/10.1177/0092070300282006

Barroso, F.G. (2008). La responsabilidad social empresarial. Un estudio de cuarenta empresas de la ciudad de Mérida, Yucatán. Contaduría y Administración. 226(2), 73-91. http://ref.scielo.org/5ph6dh

Beckmann, S. C., Christensen, A.S. \& Christensen, A.G. (2001). Myths of nature and environmentally responsible behaviours: An exploratory study. Proceedings of the 30th European Marketing Academy Conference, Bergen.

Berglind, M. \& Nakata, C. (2005). Cause- related marketing. More buck tan bang? Business Horizons, 48(5), $443-453$. https://doi.org/10.1016/j.bushor.2005.04.008

Birnbaum, M.H. (2004). Human research and data collection via the Internet. Annual Review of Psychology, 55, 803832. https://doi.org/10.1146/annurev.psych.55.090902.141601 
Bloom, P., Hoeffler, S., Keller, K. \& Basurto, E. (2006). How social-cause marketing affects consumer perceptions. MIT Sloan Management Review, 47(2). 49-55.

Boulstridge, E. \& Carrigan, M. (2000). Do consumers really care about corporate responsibility? High- lighting the attitude-behavior gap. Journal of Communication Management 4(4), 355-368. https://doi.org/10.1108/eb023532

Briseño, A., Lavín, J. \& García, F. (2011). Análisis exploratorio de la responsabilidad social empresarial y su dicotomía en las actividades sociales y ambientales de la empresa. Contaduría y Administración. 233(1), 73-90.

Carroll, A. (1991). The pyramid of social responsibility: toward the moral management of organizational stakeholders. Business Horizons, 34(4), 39-48. https://doi.org/10.1016/0007-6813(91)90005-g

Cause Marketing Forum (2015). Cause Marketing Research and Reports: IEG Sponsorship Report. Accesado el 16 de diciembre de 2015. Disponible en: http://www.causemarketingforum.com/site/c.bkLUKcOTLkK4E/b.6449067/k.71C8/ IEG_Sponsorship_Report.htm

Chang, Ch. (2008). To Donate or Not to Donate? Product Characteristics and Framing Effects of Cause-Related Marketing on Consumer Purchase Behavior. Psychology and Marketing 25(12), 1089-1110. https://doi.org/10.1002/ mar.20255

Chladek, A., Florack, A. \& Kleber, J. (2013). The Moderating Role of Numeracy in the Effectiveness of Cause-Related Marketing. En Advances in Consumer Research (41), eds. Botti, S. y Labroo, A. Duluth, Minesota: Association for Consumer Research.

Comisión de las Comunidades Europeas (2001). Libro Verde. Fomentar un marco europeo para la responsabilidad social de las empresas. Accesado el 18 de enero del 2016. Disponible en http://ec.europa.eu/transparency/regdoc/ rep/1/2001/ ES/1-2001-366-ES-F1-1.Pdf

Cone / Duke University. (2008). The 2008 Cone/Duke University Behavioral Case Study. Accesado el 16 de diciembre de 2015. Disponible en: http://www.causemarketing.biz/2008/10/the-2008-coneduke-university-behavioral-cause-study/

Edelman (2012). Edelman Good purpose Global Consumer Survey. Accesado el 16 de diciembre del 2015. Disponible en http:// www.fairtrade.travel/uploads/files/Edelman_Goodpurpose_-_Global_Consumer_Survey.pdf

Edelman, (2014). Brand purpose look ahead: Power of participation platforms. Accesado el 16 de diciembre de 2015. Disponible en http://purpose.edelman.com/2014-brand-purpose-look-ahead-power-of-participation-platforms/

Elliot, G.R. \& Cameron, R.C. (1994). Consumer perception of product quality and the country of origin effect. Journal of International Marketing, 2(2), 49-62. http://www.jstor.org/stable/25048542

Ellison, N.B., Steinfeld, C. \& Lampe, C. (2007). The benefits of Facebook "friends": Social capital and college students' use of online social network sites. Journal of Computer-Mediated Communication, 12(4), 1143-1168. https://doi.org/10.1111/j.1083-6101.2007.00367.x

FAO (16 de Diciembre del 2015). Producción y productos lácteos. Accesado el 16 de diciembre del 2015. Disponible en http:// www.fao.org/agriculture/dairy-gateway/leche-y-productos-lacteos/es/\#.VnIFEPnxyko

Folse, J.A.G., Niedrich, R.W. \& Grau, S.L. (2010). Cause-Related Marketing: The Effect of Purchase Quantity and Firm Donation Amount on Consumer Inferences and Participation Intentions. Journal of Retailing, 86(4), $295-309$. https://doi.org/10.1016/j.jretai.2010.02.005

González Hernández, E.M., Orozco Gómez, M.M. \& De la Paz Barrios. A. (2011). El Valor de la marca desde la perspectiva del consumidor. Estudio empírico sobre preferencia, lealtad y experiencia de marca en procesos de alto y bajo involucramiento de compra. Contaduría y Administración, 23 (Septiembre-Diciembre), 217-239.

Grieve, R., Witteveen, K. \& Tolan, G.A. (2014). Social media as a tool for data collection: Examining equivalence of socially value-laden constructs. Current Psychology, 33(4), 532-544. https://doi.org/10.1007/s12144-014-9227-4

Ha-Brookshire, J. E. \& Norum, P. S. (2011). Willingness to pay for socially responsible products: case of cotton apparel. Journal of Consumer Marketing, 28(5), 344-353. https://doi.org/10.1108/07363761111149992

Hall. Sheikh, S. \& Beise-Zee, R. (2011). Corporate social responsibility or cause-related marketing? The role of cause specificity of CSR. Journal of Consumer Marketing 28(1), 27-39. https://doi.org/10.1108/07363761111101921

Hayes, Andrew (2013). Introduction to Mediation, Moderation, and Conditional Process Analysis: A Regression-Based Approach (Methodology in the Social Sciences). Guilford Press, New York. https://doi.org/10.1111/jedm.12050 
Habel, J., Schons, L. M., Alavi, S. \& Wieseke, J. (2016), Warm Glow or Extra Charge? The Ambivalent Effect of Corporate Social Responsibility Activities on Customers' Perceived Price Fairness. Journal of Marketing, 80 (1), 84-105. https://doi.org/10.1509/jm.14.0389

Henderson, T. \& Arora, N. (2010). Promoting Brands Across Categories with a Social Cause: Implementing Effective Embedded Premium Programs. Journal of Marketing, 74 (November), 41-60. https://doi.org/10.1509/ jmkg.74.6.41

Keller, K. (1993). Conceptualizing, measuring and managing customer- based brand equity. Journal of Marketing 57(1), 1-22. https://doi.org/10.2307/1252054

Koschate-Fisher, N., Stefan, I. V. \& Hoyer W.D. (2012). Willingness to pay for cause-related marketing: the impact of donation amount and moderating effects. Journal of Marketing Research, 49(6), 910-927. https://doi.org/10.1509/ jmr. 10.0511

Krishna, A. \& Rajan, U. (2009). Cause Marketing: Spillover Effects of Cause-Related Products in a Product Portfolio. Management Science, 55(9), 1469-1485. https://doi.org/10.1287/mnsc.1090.1043

Kuo, A. \& Hamilton Rice, D. (2015). The impact of perceptual congruence on the effectiveness of cause-related marketing campaigns. Journal of Consumer Psychology, 25(1), 78-88. https://doi.org/10.1016/j.jcps.2014.06.002

Lafferty, B. A. \& Goldsmith, R. E. (2005). Cause-brand alliance: does the cause help the brand or does the brand help the cause? Journal of Business Research, 58(4). 423-429. https://doi.org/10.1016/j.jbusres.2003.07.001

Lafferty, B. A., Goldsmith, R. E. \& Hult, G. T. M. (2004). The impact of the alliance on the partners: A look at cause-brand alliances. Psychology \& Marketing, 21(7), 509-531. https://doi.org/10.1002/mar.20017

León, F. (2008). La percepción de la responsabilidad social empresarial por parte del consumidor. Visión Gerencial. 1. 83-95.

Lichtenstein, D.R., Drumwright, M.E. \& Braig, B.M. (2004). The Effect of Corporate Social Responsibility on Customer Donations to Corporate-Supported Nonprofits. Journal of Marketing, 68 (September), 16-32. https://doi. org/10.1509/jmkg.68.4.16.42726

Lusk, J. L. \& Shogren, J. F. (2007). Experimental auctions. Methods and applications in economic and marketing research. Cambridge: Cambridge University Press. https://doi.org/10.1017/cbo9780511611261

Marin, L., Ruiz S. \& Rubio, A. (2009). The role of identity salience in effects of corporate social responsibility on consumer behavior. Journal of Business Ethics, 84(65-78). https://doi.org/10.1007/s10551-008-9673-8

McWilliams, A., Siegel, D. S. \& Wright, P.M. (2006). Corporate Social Responsibility: Strategic Implications. Journal of Management Studies, 43(1), 1-18. https://doi.org/10.1111/j.1467-6486.2006.00580.x

Mohr, L.A. \& Webb, D.J. (2005). The effects of corporate social responsibility and Price on Consumer Responses. Journal of Consumer Affairs, 39(1), 121-146. https://doi.org/10.1111/j.1745-6606.2005.00006.x

Petkus, E. \& Woodruff, R. (1992). A model of the socially responsible decision-making process in marketing: linking decision makers and stakeholders. En Chris Allen et al. (Eds.), Proceedings of the winter 1992 American Marketing Association (154-161). Chicago: American Marketing Association.

Piercy, N.F. \& Lane, N. (2009). Corporate social responsibility: Impacts on strategic marketing and customer value. The Marketing Review, 9(4), 335-360. https://doi.org/10.1362/146934709x479917

Popkowski-Leszczyc, P.T.L. \& Wong, L.T. (2010). The Effectiveness of Donation Promises in Charity Auctions as a Cause-Related Marketing Strategy (June 30, 2010). Disponible en SSRN:http://ssrn.com/abstract=1734505 o http:// dx.doi.org/10.2139/ssrn.1734505

Pracejus, J.W. Olsen, G.D. \& Brown, N.R. (2003). On the Prevalence and Impact of Vague Quantifiers in the Advertising of Cause-Related Marketing (CRM). Journal of Advertising, 32(4), 19-28. https://doi.org/10.1080/009133 67.2003.10639146

Raajpoot, N., Javed, J. \& Koh, K. (2008). Application of Taguchi design to retail service. International Journal of Commerce and Management, 18(2), 184 - 199. https://doi.org/10.1108/10569210810895258

Robinson, S. R., Irmak, C. \& Jayachandran, S. (2012). Choice of Cause in Cause-Related Marketing. Journal of Marketing, 76 (July), 126-139. https://doi.org/10.1509/jm.09.0589

Schiffman, L. \& Kanuk, L. (2005). Comportamiento del consumidor. 8a Ed. México: Pearson Prentice

Simon, H. A. (1959). Theories of decision-making in economics and behavioral science. The American Economic Review, 49(3), 253-283. http://www.jstor.org/stable/1809901 

http://dx.doi.org/10.22201/fca.24488410e.2018.1013

Smith, N. Hall. Sheikh, S. \& Beise-Zee, R. (2011). Corporate social responsibility or cause-related marketing? The role of cause specificity of CSR. Journal of Consumer Marketing 28(1), 27-39. https://doi. org/10.1108/07363761111101921

Subrahmanyan, S. (2004). Effects of Price Premium and Product Type on the Choice of Cause- Related Brands: A Singapore Perspective. Journal of Product \& Brand Management, 13(2), 116-124. https://doi. org/10.1108/10610420410529744

Tian, Z., Wang, R. \& Yang, W. (2011). Consumer Responses to Corporate Social Responsibility (CSR) in China. Journal of Business Ethics 101(2), 197-212. https://doi.org/10.1007/s10551-010-0716-6

TNS research international, (2011). Green study 2011. Accesado el 16 de diciembre de 2015. Disponible en http:// www.tns-ri.com. mx/pdf/Green_reporte_2011.pf

TNS research international, (2013). The green disconnect. Consumer attitudes and behaviours in the EU. Accesado el 16 de diciembre de 2015. Disponible en: http://www.tnsglobal.com/sites/default/files/whitepaper/TNSUK_Green2013Oct28.pdf.

Van den Brink, D., Oderken Schöreder, G. \& Powels, P. (2006). The effect of strategic and tactical cause-related marketing on consumers' brand loyalty. Journal of Consumer Marketing, 23(1), 15-25. https://doi. org/10.1108/07363760610641127

Varadarajan, P.R. \& Menon, A. (1988). Cause- related marketing: A coalignment of marketing strategy and corporate philanthropy. The Journal of Marketing, 52(3), 58-74. https://doi.org/10.2307/1251450

Veenhoven, R. (2008). Green consumerism: approaches in country experiences. The Icfai University Press, Hyderabad, India, p.39-68.

Vives, A. (2008). ¿Es nuestra responsabilidad? Harvard Business Review.86(4), 50-54.

We are social (2015). Digital, social \& mobile in 2015. We are social's compendium of global digital statistics. Recuperado el 28 de abril del 2016. Disponible en http://wearesocial.sg/blog/2015/01/digital-social-mobile-2015/

WFA, (2013). How important is it for brands to have a "purpose"? World Federation of Advertisers. Recuperado el 16 de abril del 2016. Disponible en http://www.wfanet.org/en/global-news/how-important-is-it-for-brands-to-have-a-u201cpurposeu201d

Williams, E. (2011). Responsible consumers and stakeholder marketing: building a virtuous circle of social responsibility. Universia Business Review, 30, 68-78.

Winterich, K.P. \& Barone, M.J. (2011). Warm Glow or Cold, Hard Cash? Social Identity Effects on Consumer Choice for Donation Versus Discount Promotions. Journal of Marketing Research, 48(5), 855-868. https://doi. org/10.1509/jmkr.48.5.855

Wooldridge, J. M. (2009). Introductory econometrics: A modern approach, México: Thomson/South-Western.

\section{Anexo 1}

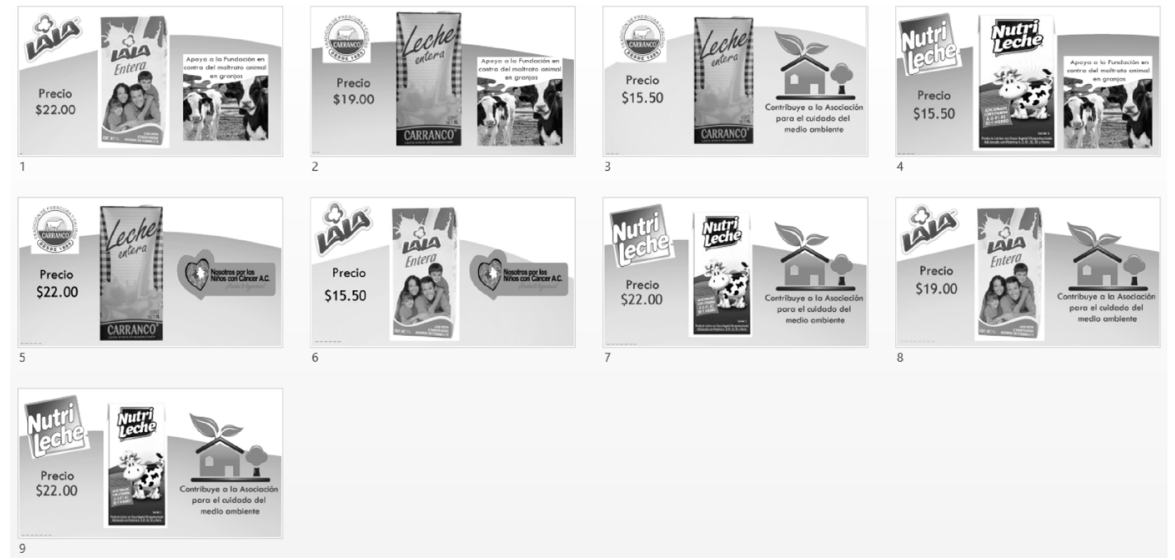

